\title{
In vivo Anti-Cancer Effects of Resveratrol Mediated by NK Cell Activation
}

\author{
Yoojin Lee ${ }^{a, b}$ Heewook Shin ${ }^{a, b}$ Jongsun Kim ${ }^{a}$ \\ aDepartment of Microbiology and Institute for Immunology and Immunological Diseases, Yonsei University College \\ of Medicine, Seoul, Republic of Korea; ${ }^{b}$ Brain Korea 21 PLUS Project for Medical Science, Yonsei University College of \\ Medicine, Seoul, Republic of Korea
}

\section{Keywords}

Natural killer cell · Resveratrol · Interferon-gamma · Natural killer cytotoxicity $\cdot$ Immunotherapy

\begin{abstract}
Natural killer (NK) cells are innate immune lymphocytes that play an important role in anti-viral and anti-tumour immune responses. Several cancer immunotherapy approaches targeting NK cells are currently in clinical or preclinical development. Here, we aimed to find food nutrients that activate NK cells and determine their usefulness as candidates for anticancer and anti-metastatic drugs. Resveratrol appeared to activate NK cells most effectively among the substances tested and synergistically increased IFN- $\gamma$ secretion and NK cell cytotoxicity with interleukin-2 (IL-2). CD107a, NKp30, and NKG2D expression levels were upregulated on the surface of NK cells upon treatment with resveratrol in combination with IL-2 compared with treatment with IL-2 alone. Moreover, NK cell activity in human and mouse whole blood was enhanced upon treatment with resveratrol. Most importantly, administration of resveratrol effectively inhibited tumour growth and metastasis in mice. In conclusion, we suggest that resveratrol may represent a candidate anti-cancer drug that acts by activating NK cells in vivo.
\end{abstract}

(C) 2020 The Author(s)

Published by S. Karger AG, Basel

\section{Introduction}

Natural killer (NK) cells are innate immune lymphocytes accounting for about $15 \%$ of all circulating lymphocytes that spontaneously kill cells deemed to be dangerous to the host in the absence of prior sensitization; they thus are presumed to be key effectors in cancer immunosurveillance, transplantation rejection, and early viral immunity [1-3]. Recently, it was reported that physical activity and metabolic status may alter the immune system and that physical inactivity and metabolic abnormalities are associated with reduced NK cell activity [4]. Further, NK cells are potent killers of malignant cells and are particularly important in limiting tumour metastasis $[5,6]$. NK cells can inhibit tumour growth by direct cytotoxicity through the release of cytolytic granules, by secretion of cytokines such as interferon-gamma (IFN- $\gamma$ ), or indirectly by the orchestration of anti-tumour immune responses [7]. NK cells are activated by Th1-type cytokines, such as interleukin (IL)-2, $-12,-15$, or -18 , natural cytotoxicity receptors, and NKG2D [8-10]. When NK cells are activated, they secrete large amounts of IFN- $\gamma$, which is essential in viral and tumour clearance and activates the signal transducers and activators of transcription (STAT) signalling pathway in target cells, leading to the improved control of infection $[11,12]$. IFN- $\gamma$ production by NK cells restricts tumour angiogenesis, stimulates adaptive
(C) 2020 The Author(s)

Published by S. Karger AG, Basel

This article is licensed under the Creative Commons AttributionNonCommercial-NoDerivatives 4.0 International License (CC BYNC-ND) (http://www.karger.com/Services/OpenAccessLicense) Usage and distribution for commercial purposes as well as any distribution of modified material requires written permission.
Jongsun Kim

Department of Microbiology and Institute for Immunology and Immunological Diseases, Yonsei University College of Medicine

50-1 Yonsei-ro, Seodaemun-gu, Seoul 03722 (Republic of Korea) jkim63@yuhs.ac 
immunity, and helps shape T-cell responses in lymph nodes [13].

Third-generation immunotherapy represents a new paradigm for cancer treatment [14]. As the focus of treatment has shifted from the tumour itself to the host's immune system, cancer immunotherapies activating the body's immune system to fight cancer cells have been developed. Most immunotherapeutic strategies focus on enhancing T-cell responses; however, there has been recent interest in using the relatively underexplored NK cell compartment for therapeutic interventions [15-17]. Moreover, it is becoming clear that some of the clinical approaches originally developed to increase T-cell cytotoxicity may also activate NK cells [16].

In some clinical studies, the use of a low dose of IL-2, alone or in combination with other agents (such as lowdose IL-2 and IFN- $\alpha$ ), results in similar clinical response rates and survival probabilities as the use of high doses of IL-2 alone [18]. Unfortunately, cytokines and other small molecules secreted by IL-2-activated effector cells induce toxicity, which offsets the efficacy of IL-2. To overcome this limitation, studies of therapies involving new cytokines are underway, including the use of IL-12 alone or in combination with IL-2 $[19,20]$. In the last decade, the field of nutritional immunology has grown steadily. We hypothesized that, in addition to cytokines, there may be substances among natural compounds with the ability to activate NK cells [21].

Here, we screened several compounds including antioxidants, vitamins, and food ingredients to identify compounds that can activate NK cells. Furthermore, we demonstrated the potential of one of these compounds, resveratrol, as an anti-cancer and anti-metastatic drug candidate, which may act via NK cell activation. Overall, our finding enlightens the novel function of resveratrol as an NK booster, which can be the potential adjuvant for immunotherapeutics in cancers.

\section{Materials and Methods}

\section{Cell Lines and Cell Culture}

The human NK cell line NK92 (ATCC ${ }^{\circledR}$ CRL-2407 ${ }^{\mathrm{TM}}$ ) was maintained in the minimum essential medium- $\alpha$ (MEM- $\alpha$ ) (Gibco, New York, NY, USA) supplemented with $12.5 \%$ heat-inactivated foetal bovine serum (FBS; Gibco), 12.5\% heat-inactivated horse serum (Gibco), 0.2-mM myo-inositol (Sigma-Aldrich, St. Louis, MO, USA), 0.1-mM 2-mercaptoethanol (Sigma-Aldrich), 0.02-mM folic acid (Sigma-Aldrich), 1\% penicillin/streptomycin (WelGENE, Gyeongsansi, Korea), and $5 \mathrm{ng} / \mathrm{mL}$ IL-2 (ATGen, Sungnamsi, Korea). Human erythroleukemia cell line K562 (ATCC ${ }^{\circledR}$ CCL-243 ${ }^{\mathrm{TM}}$ ) was maintained in the RPMI 1640 medium (Gibco) supplemented with $10 \%$ FBS and $1 \%$ penicillin/streptomycin. Mouse melanoma cell line B16F10 (ATCC ${ }^{\circledR}$ CRL-6475 ${ }^{\mathrm{TM}}$ ) was maintained in Dulbecco's modified Eagle's medium (DMEM) (Gibco) supplemented with $10 \% \mathrm{FBS}$ and $1 \%$ penicillin/streptomycin.

\section{Antibodies and Reagents}

Resveratrol, 3-amino-1-propanesulfonic acid (3-APS), glutathione, taurine, glycine, alanine, $\beta$-alanine, $\beta$-carotene, retinoic acid, retinol, retinal, tazarotene, vitamin B1, vitamin B12, vitamin D3, 2,3-butandiol, quercetin, triacetyl resveratrol, and piceatannol were purchased from Sigma-Aldrich. Lycopene and catechin were purchased from Santa Cruz Biotechnology (Santa Cruz, CA, USA). Resveratrol-3-O-glucuronide was purchased from Cayman Chemical (Ann Arbor, MI, USA). Resveratrol-4'-glucuronide was purchased from Spibio (Montigny Le Bretonneux, France). Resveratrol-trisulphate was purchased from Carbosynth (Berkshire, UK). For flow cytometry, PE-conjugated anti-human CD107a antibody (555801) and APC-conjugated anti-human CD56 antibody (555518) were purchased from BD Pharmingen (San Diego, CA, USA). BV421-conjugated anti-human CD3 antibody (300434), APC-Cy7-conjugated anti-human CD16 antibody (302017), PEconjugated anti-human NKG2D antibody (320806), PE-conjugated anti-human NKp30 antibody (325208), PE-conjugated anti-human NKp44 antibody (325108), PE-conjugated anti-human NKp46 antibody (331908), BV650-conjugated anti-mouse TCR $\beta$ antibody (109251), PE-conjugated anti-mouse NK1.1 antibody (108708), PerCP-Cy5.5-conjugated anti-mouse IFN- $\gamma$ antibody (505822), and PE-Cy7-conjugated anti-mouse CD107a antibody (121619) were purchased from BioLegend (San Diego, CA, USA).

\section{Cell Proliferation Assay}

Cell proliferation was measured using the CCK- 8 assay kit (Dojindo Laboratories, Kumamoto, Japan). NK92 cells $\left(1 \times 10^{4}\right.$ cells/well) $(100 \mu \mathrm{L})$ were dispensed in triplicate into a 96 -well flatbottom plate and incubated for $4 \mathrm{~h}$. After incubation, $10 \mu \mathrm{L}$ of the CCK-8 solution was added to each well. After $4 \mathrm{~h}$, absorbance was measured at $450 \mathrm{~nm}$ using a microplate reader.

\section{ELISA}

An IFN- $\gamma$ ELISA set was purchased from BD Biosciences (San Diego, CA, USA). NK92 cells $\left(5 \times 10^{4}\right.$ cells/well $)$ were dispensed into a 24-well plate and incubated for $36 \mathrm{~h}$. Then, the supernatants were harvested and dispensed in triplicate into a 96-well microplate pre-coated with capture antibody. After incubation for $2 \mathrm{~h}$, each well was washed with the washing buffer $(0.05 \%$ Tween 20 in PBS, $\mathrm{pH}$ 7.4), followed by the addition of HRP-conjugated detection antibody and incubation for $1 \mathrm{~h}$. The substrate solution $3,3^{\prime}, 5,5^{\prime}$-tetramethylbenzidine (TMB) was added under protection from light. Absorbance was measured at $450 \mathrm{~nm}$ using a microplate reader.

\section{CD107a Degranulation Assay}

NK92 cells were treated with or without resveratrol and incubated for $36 \mathrm{~h}$. After incubation, NK92 cells $\left(2 \times 10^{4}\right.$ cells/well $)$ were dispensed into a 96-well round-bottom plate and incubated with an equal number of K562 target cells. Then, anti-CD107a antibody was added, and the plate was incubated for $4 \mathrm{~h}$. Cells were harvested and stained with anti-CD56 antibody for $30 \mathrm{~min}$ on ice. After 2-3 washes with PBS containing $1 \%$ FBS, fluorescence was measured using a BD LSR II flow cytometer (BD Biosciences). PBMCs were treated with or without resveratrol for $24 \mathrm{~h}$ in the 
presence of IL-2. After incubation, PBMCs were incubated with K562 cells (5:1 ratio) for $4 \mathrm{~h}$. Cells were harvested and stained with anti-CD3,-CD16, and -CD56 antibody in the presence of fixable live/dead stain (Invitrogen) for $30 \mathrm{~min}$ on ice. After 2-3 washes with PBS containing $1 \%$ FBS, fluorescence was measured using a BD LSR II flow cytometer (BD Biosciences).

\section{Flow Cytometry}

NK92 cells $\left(5 \times 10^{4}\right.$ cells $\left./ 500 \mu \mathrm{L}\right)$ were incubated with or without resveratrol. After incubation, cells were washed twice with PBS containing $1 \%$ FBS and stained with fluorochrome-conjugated antibodies in PBS containing 1\% FBS for 30 min on ice in the dark. The cells were washed twice, and then fluorescence was measured using BD LSR II.

\section{Real-Time PCR Analysis}

Total RNA was extracted from NK92 cells using TRIzol (Life Technologies, Carlsbad, CA, USA), and cDNA was synthesized using the SensiFAST cDNA synthesis kit (Bioline, Taunton, MA, USA) according to the manufacturer's instructions. Synthesized cDNAs were used as templates for subsequent PCR amplification of the IFNG, NKp30 isoforms, and GAPDH genes. The following primers were used:

1. IFNG, $5^{\prime}$-GTCCAACGCAAAGCAATACA- $3^{\prime}$ and $5^{\prime}$-CTCTTCGACCTCGAAACAGC- $3^{\prime}$

2. NKp30a, $5^{\prime}$-GGTGGTGGAGAAAGAACATC- $3^{\prime}$ and $5^{\prime}$-CTTTCCAGGTCAGACATTTGC- $3^{\prime}$

3. NKp30b, $5^{\prime}$-GGTGGTGGAGAAAGAACATC- ${ }^{\prime}$ and $5^{\prime}$-GAGAGTAGATTTGGCATATTTGC-3'

4. NKp30c, $5^{\prime}$-GGTGGTGGAGAAAGAACATC- ${ }^{\prime}$ and $5^{\prime}$-CATGTGACAGTGGCATTTGC- ${ }^{\prime}$

5. GAPDH, $5^{\prime}$-CAGCCTCAAGATCATCAGCA- $3^{\prime}$ and $5^{\prime}$-GTCTTCTGGGTGGCAGTGAT- $3^{\prime}$

Quantitative real-time RT-PCR was carried out using a KAPA SYBR FAST qPCR kit (KAPA Biosystems, Wilmington, MA, USA), and amplification was performed on an ABI Prism StepOnePlus ${ }^{\mathrm{TM}}$ detection system (Applied Biosystems, Foster City, CA, USA) according to the conditions recommended by the manufacturer. The experiments were performed in triplicate, and results were normalized to the expression of GAPDH. The relative expression levels of the target genes were calculated by the $2^{-\Delta \Delta \mathrm{Ct}}$ method.

\section{Cytotoxicity Assay}

The calcein-AM (Invitrogen, Carlsbad, CA, USA) assay was used to measure NK cell cytotoxicity against target cells. CalceinAM was added to target cells to a final concentration of $2 \mu \mathrm{M}$ and incubated for $30 \mathrm{~min}$ at $37^{\circ} \mathrm{C}$ and $5 \% \mathrm{CO}_{2}$. Calcein-AM-labelled target cells were washed with PBS twice, and then $1 \times 10^{4}$ cells were dispensed in quadruplicate into a 96 -well round-bottom plate. NK cells were added at different effector:target (E:T) ratios and cocultured for $4 \mathrm{~h}$. The calcein-AM released from lysed target cells was measured with a spectrophotometer (ex: $485 \mathrm{~nm} / \mathrm{em}: 535 \mathrm{~nm}$ ). Total specific lysis was calculated by the following formula:

$$
\text { Specific cytotoxicity }(\%)=\frac{(\text { experimental release }- \text { spontaneous release })}{(\text { maximum release }- \text { spontaneous release })} \times 100
$$

\section{Ex vivo NK Cell Activity Assay}

Blood was obtained from healthy donors after acquiring institutional review board approval and informed consent (No. 4-2019-
0007). Animal experiments were approved by the Yonsei University Health System Institutional Animal Care and Use Committee (YUHS-IACUC, No. 2017-0203).

For the human NK cell activity assay, $1 \mathrm{~mL}$ of whole blood was incubated with the activator (provided as part of the kit) for $24 \mathrm{~h}$ at $37^{\circ} \mathrm{C}$ under $5 \% \mathrm{CO}_{2}(n=13)$. For the analysis of mouse NK cell activity, $100 \mu \mathrm{L}$ of whole blood was incubated with $30 \mu \mathrm{L}$ activator for $24 \mathrm{~h}$ at $37^{\circ} \mathrm{C}$ (tumour-naïve or tumour-bearing mouse model, $n=8$ ). After incubation, the supernatant was collected, and the released IFN- $\gamma$ was measured using the murine NK Vue kit (ATGen). Standards and samples were then incubated in an anti-human (or murine) IFN- $\gamma$ antibody-coated plate for $2 \mathrm{~h}(1 \mathrm{~h}$ for murine) at $20-25^{\circ} \mathrm{C}$. After incubation, each well was washed 4 times before the addition of HRP-conjugated detection antibody and incubated for $1 \mathrm{~h}$. Each well was washed 7 times (4 times for murine), and then the TMB substrate solution was added in the dark. After incubation for $30 \mathrm{~min}$, the stop solution was added, and absorbance was measured at $450 \mathrm{~nm}$.

\section{Animal Studies}

Animal experiments were approved by the Yonsei University Health System Institutional Animal Care and Use Committee (YUHS-IACUC, No. 2017-0203). Specific pathogen-free female C57BL/6 mice, 7 weeks of age, were purchased from Orient Bio (Seoul, Korea). Mice were maintained in a temperature-controlled, air-conditioned animal house under a 12/12-h light/dark cycle and received food and water ad libitum. Animals were cared for and treated in accordance with the procedures outlined in the Guide for the Care and Use of Laboratory Animals (National Research Council, WA, DC, USA). B16F10 cells were used between passages 4 and 5 .

For the anti-tumour assay, B16F10 cells $\left(5 \times 10^{5}\right.$ cells $)$ in $0.2 \mathrm{~mL}$ of PBS were injected subcutaneously (s.c.) into the right flanks of mice on day 0 . On day 18 , the mice were sacrificed, and the tumours were dissected. The activity of NK cells isolated from the tumour and draining lymph nodes was evaluated by flow cytometry. Tumour volume was calculated for each mouse by measuring the length $(L)$, width $(W)$, and height/diameter $(H)$ of approximately ellipsoid tumours using a caliper, according to the following formula:

Tumor volume $\left(\mathrm{mm}^{3}\right)=L \times W \times H \times 0.52$

For the pulmonary tumour metastasis assay, B16F10 cells at a concentration of $1 \times 10^{5}$ cells in $0.1 \mathrm{~mL}$ of PBS were injected intravenously (i.v.) into the tail veins of mice on day 0 . On day 18 , the mice were sacrificed, and the lungs were extracted. The melanoma foci on the lung surface were counted macroscopically. The activity of NK cells isolated from the lungs was evaluated by flow cytometry.

To evaluate the activity of resveratrol, $0.5 \mathrm{mg} / \mathrm{kg}$ of resveratrol in $0.1 \mathrm{~mL}$ of PBS was i.v. injected a total of 6 times, on days $-2,0$, $2,4,6$, and $8(n=10$ or 7$)$.

\section{NK Cell Depletion Model}

To deplete NK cells, $25 \mu \mathrm{g}$ anti-NK1.1 monoclonal antibody (PK136) or control mouse IgG2a (both from Bio-X-Cell) was injected intraperitoneally (i.p.) on days $-3,2$, and 8. B16F10 cells $\left(5 \times 10^{5}\right.$ cells) in $0.2 \mathrm{~mL}$ of PBS were injected subcutaneously into the right flanks of mice on day 0 . On day 18 , the mice were sacrificed, and the tumours were dissected, and tumour volume was calculated.

\section{Statistical Analysis}

Statistical analysis was performed with GraphPad Prism software (GraphPad Software, San Diego, CA, USA). Data are ex-
Lee/Shin/Kim 


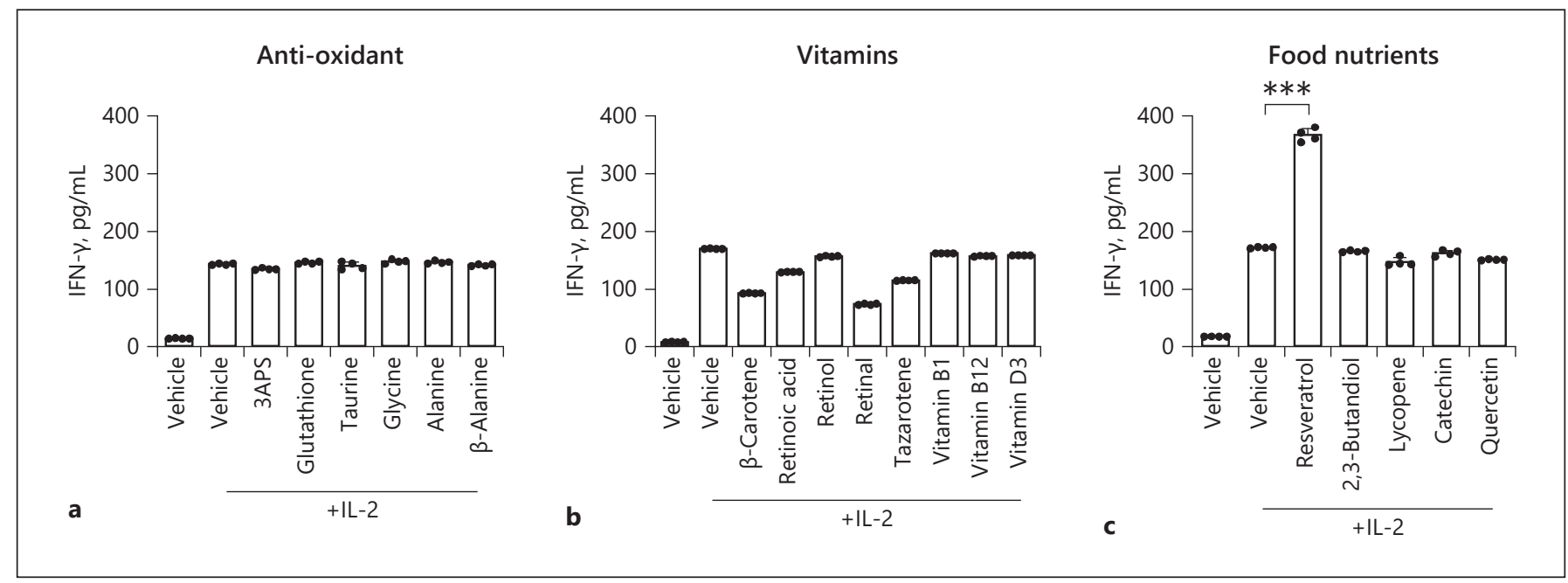

Fig. 1. Screening of NK cell-activating molecules. a-c Comparisons of IFN- $\gamma$ secretion in NK92 cells treated with resveratrol and other compounds (with $5 \mathrm{ng} / \mathrm{mL}$ of IL-2) for $36 \mathrm{~h}$. IFN- $\gamma$ secretion was measured by ELISA. Data are shown as mean \pm SEM of 4 independent experiments. Asterisks indicate statistical significance by one-way ANOVA: ${ }^{*} p<0.05 ;{ }^{* *} p<0.01 ;{ }^{* * *} p<0.001$; ns, not significant $(p>0.05)$. IL-2, interleukin-2; IFN- $\gamma$, interferongamma.

pressed as means \pm standard error of the mean (SEM). One- or two-way ANOVA was used to compare distributions between groups. $p<0.05$ was considered statistically significant.

\section{Results}

\section{Screening of NK Cell-Activating Molecules}

To identify a non-cytokine natural substance capable of activating NK cells, several compounds, including known antioxidants (Fig. 1a), vitamin-based substances (Fig. 1b), and food ingredients (Fig. 1c), were tested for their ability to activate NK92 cells. In this study, the vehicle is the solvent control which contains the same amount of ethanol used to dissolve each compound. Since NK cells require IL-2 to proliferate, the experiment was conducted in the presence of IL-2. Most of the compounds appeared to have no effect on NK cell viability when added at a concentration of up to $20 \mu \mathrm{M}$, whereas retinoic acid and retinal slightly inhibited cell proliferation (data not shown). IFN- $\gamma$ secretion was next measured to determine whether NK cells were activated by these substances. Interestingly, while most substances appeared to have no effect on IFN- $\gamma$ secretion, only resveratrol significantly increased IL-2-induced IFN- $\gamma$ secretion about 2 -fold compared to that of IL-2 treatment alone, suggesting potential roles of resveratrol in activating NK cells (Fig. 1).

Resveratrol Activates NK Cells in vivo

\section{Resveratrol Increased IL-2-Induced IFN- $\gamma$ Secretion from NK Cells}

As mentioned above, among the antioxidants, vitamins, and food nutrients tested, only resveratrol appeared to increase the IFN- $\gamma$ secretion from NK cells (Fig. 1). Moreover, as shown in Figure 2a, b, resveratrol did not significantly affect NK cell proliferation but increased IFN- $\gamma$ secretion in a dose-dependent manner. Particularly, at a resveratrol concentration of $20 \mu \mathrm{M}$, IFN- $\gamma$ secretion was increased by $>50 \%$ compared to that of IL2-alone-treated cells (Fig. 2b). Next, we measured NK cell proliferation and IFN- $\gamma$ secretion after treatment with resveratrol with or without IL-2 to investigate whether resveratrol is effective as a replacement for IL-2 or has synergistic effects in combination with IL-2. As shown in Figure $2 c, d$, resveratrol had no effect in the absence of IL-2. Moreover, in the presence of IL-2, NK cell proliferation was not affected by resveratrol (Fig. 2c). While IFN- $\gamma$ secretion was not altered for up to $12 \mathrm{~h}$ after treatment, it thereafter increased by about $40 \%$ or more compared to that observed in the group treated with IL-2 alone (Fig. 2d). We also confirmed by real-time PCR that the mRNA level of IFNG was upregulated by resveratrol treatment in the presence, but not the absence, of IL-2 (Fig. 2e). Altogether, these results indicate that resveratrol has synergistic effects in combination with IL-2 in activating NK cells. 

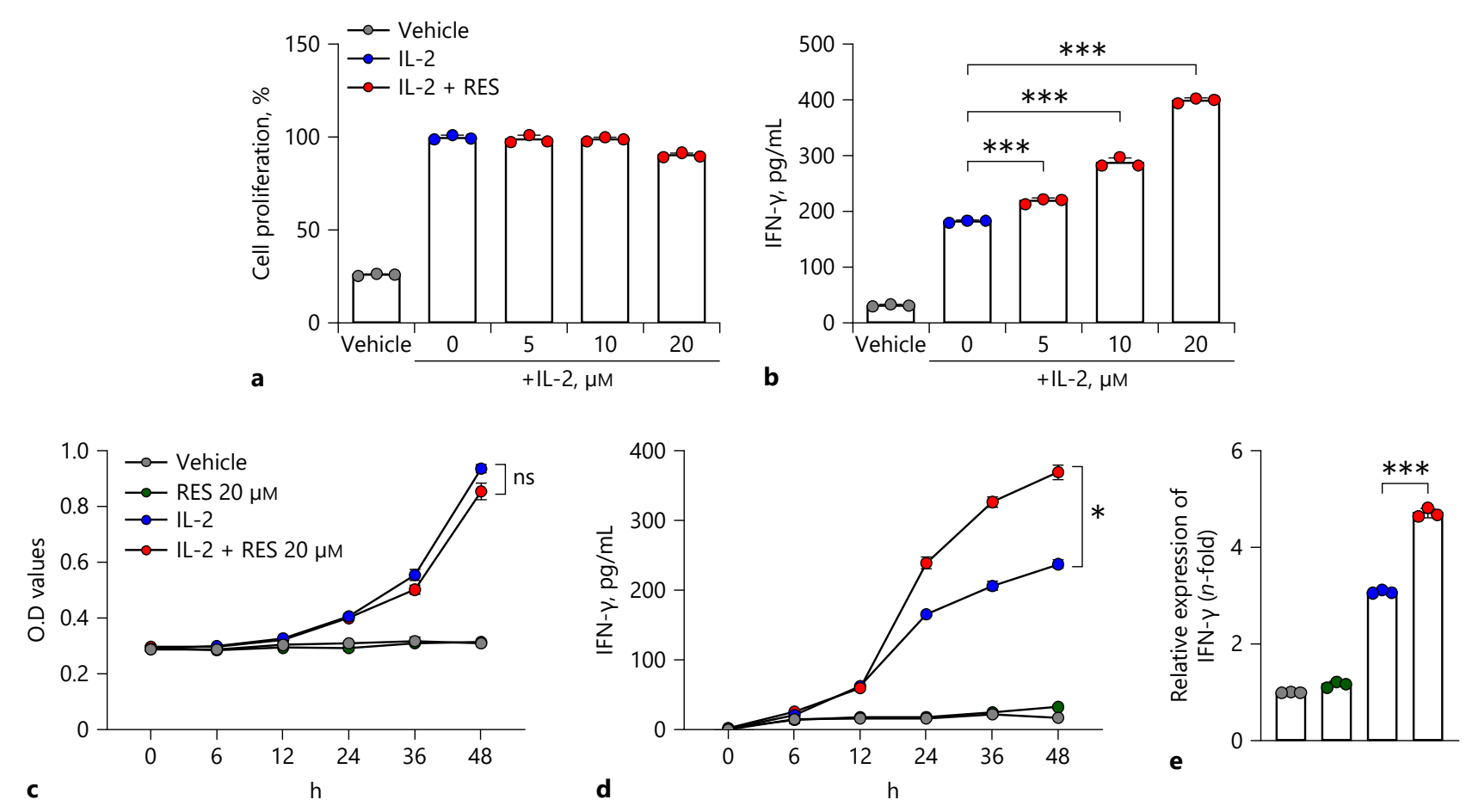

Fig. 2. Resveratrol increased IL-2-induced IFN- $\gamma$ secretion from NK cells. a, b NK92 cells were treated with 0-20 $\mu \mathrm{M}$ resveratrol in the presence of $5 \mathrm{ng} / \mathrm{mL}$ IL-2 for $36 \mathrm{~h}$. c, d NK92 cells were treated with $20 \mu \mathrm{M}$ resveratrol for $0-48 \mathrm{~h}$ with or without $5 \mathrm{ng} /$ mL IL-2. e The mRNA levels of IFNG in NK92 cells after treatment of resveratrol for $24 \mathrm{~h}$ were measured by real-time PCR and

\section{Effects of Resveratrol on NK Cell Activity and}

\section{Activating Receptor Expression}

We next examined the effects of resveratrol on NK cell degranulation activity by measuring CD107a expression as a functional marker of NK cell activation [22]. NK cells were co-incubated with K562 target cells, which do not express major histocompatibility complex (MHC) class I molecules and are susceptible to NK cell-mediated killing [23]. This was followed by analysis of surface levels of CD107a by flow cytometry. As shown in Figure 3a, after NK cells were pretreated with IL-2, NK cells expressing CD107a accounted for $18.3 \pm 0.23 \%$ of all $\mathrm{CD}^{2} 6^{+} \mathrm{NK}$ cells. CD107a expression on the surface of NK cells pretreated with resveratrol and IL-2 increased 1.8-fold compared to that on the surface of IL-2-alone-pretreated cells, resulting in $32.9 \pm 2.59 \%$ of $\mathrm{CD}^{2} 6^{+} \mathrm{NK}$ cells expressing CD107a. Next, we measured the effects of resveratrol on the expression of NK cell-activating receptors which have central roles for NK cell cytotoxicity. As shown in Figure calculated by the $\Delta \Delta \mathrm{CT}$ method. Gapdh was used as an internal control. Data are shown as mean \pm SEM of 3 independent experiments. Asterisks indicate statistical significance by one- or twoway ANOVA: ${ }^{*} p<0.05 ;{ }^{* *} p<0.01 ;{ }^{* *} p<0.001$; ns, not significant ( $p>0.05)$. RES, resveratrol; IL-2, interleukin-2; IFN- $\gamma$, interferongamma.

$3 \mathrm{~b}$, the expression of NK cell-activating receptors was significantly increased by resveratrol more than IL-2 alone. Since a previous study has shown immunomodulatory functions of NKp30 depending on its isoforms [24], we analyzed the change in the expression level of NKp30 isoforms upon IL-2 with or without resveratrol treatment. While resveratrol did not affect the expression of NKp30c, known as an inhibitory Nkp30 isoform [24], the activating isoforms including NKp30a as well as NKp30b were significantly increased by resveratrol (Fig. 3c). Altogether, these results suggest that resveratrol might enhance overall cytotoxicity of NK cells. Hence, we examined the effect of resveratrol on the cytotoxicity of NK cells. Consistent with previous studies, IL-2 treatment significantly enhanced the killing activity of NK cells (Fig. 3d). Intriguingly, co-treatment of resveratrol together with IL-2 further heightened NK cytotoxicity by $21.3 \%$ compared with IL-2-treated cells at 1:1 ratio, indicating the increment of NK cytotoxicity by resveratrol (Fig. 3d). 


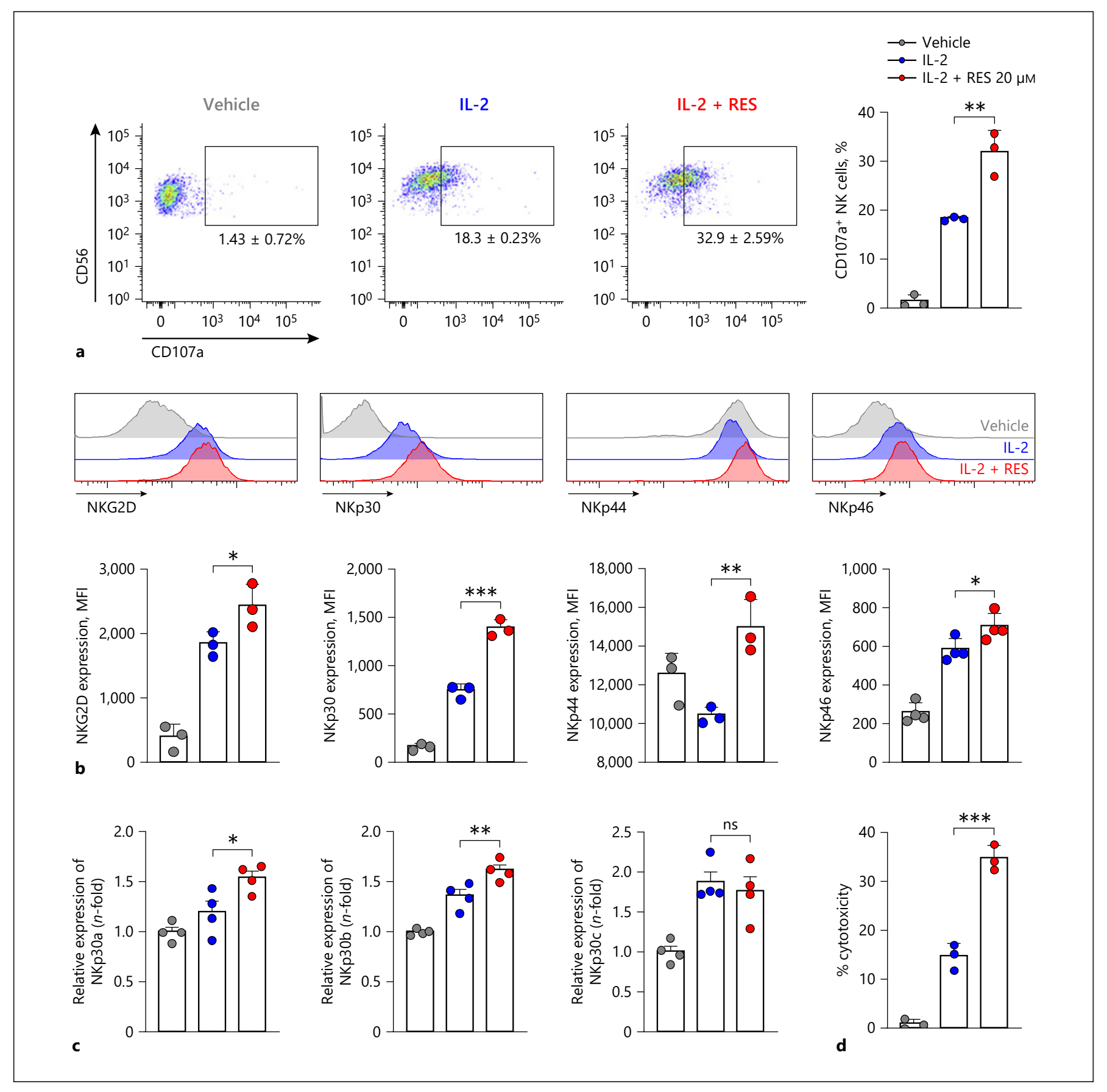

Fig. 3. Effects of resveratrol on NK cell activity and activating receptor expression. a CD107a expression on the surface of NK92 cells after resveratrol treatment was measured by flow cytometry. NK92 cells were treated with $20 \mu \mathrm{M}$ resveratrol for $36 \mathrm{~h}$, followed by co-culture with K562 target cells at a 1:1 ratio for $4 \mathrm{~h}$. Quantitative analysis showing the percentages of $\mathrm{CD} 56^{+} \mathrm{CD} 107 \mathrm{a}^{+}$cells. Data are shown as mean \pm SEM of 3 independent experiments. b Expression of natural cytotoxicity receptors and NKG2D was measured by flow cytometry. NK92 cells were treated with $20 \mu \mathrm{M}$ resveratrol for $36 \mathrm{~h}$. Data are shown as mean \pm SEM of 3 independent experiments. $\mathbf{c}$ The mRNA levels of NKp30 isoforms in NK92 cells after treatment of resveratrol for $6 \mathrm{~h}$ were measured by realtime PCR and calculated by the $\Delta \Delta C T$ method. Gapdh was used as an internal control. Data are shown as mean \pm SEM of 4 independent experiments. d NK cell cytotoxicity was determined using calcein-AM. NK92 effector (E) cells were treated with $20 \mu \mathrm{M}$ resveratrol for $36 \mathrm{~h}$, followed by incubation with K562 target $(\mathrm{T})$ cells at a 1:1 ratio for $4 \mathrm{~h}$. Data are shown as mean \pm SEM of 3 independent experiments. Asterisks indicate statistical significance by oneway ANOVA: ${ }^{*} p<0.05 ;{ }^{* *} p<0.01 ;{ }^{* * *} p<0.001 ;$ ns, not significant $(p>0.05)$. RES, resveratrol; IL-2, interleukin-2; NK cells, natural killer cells. 


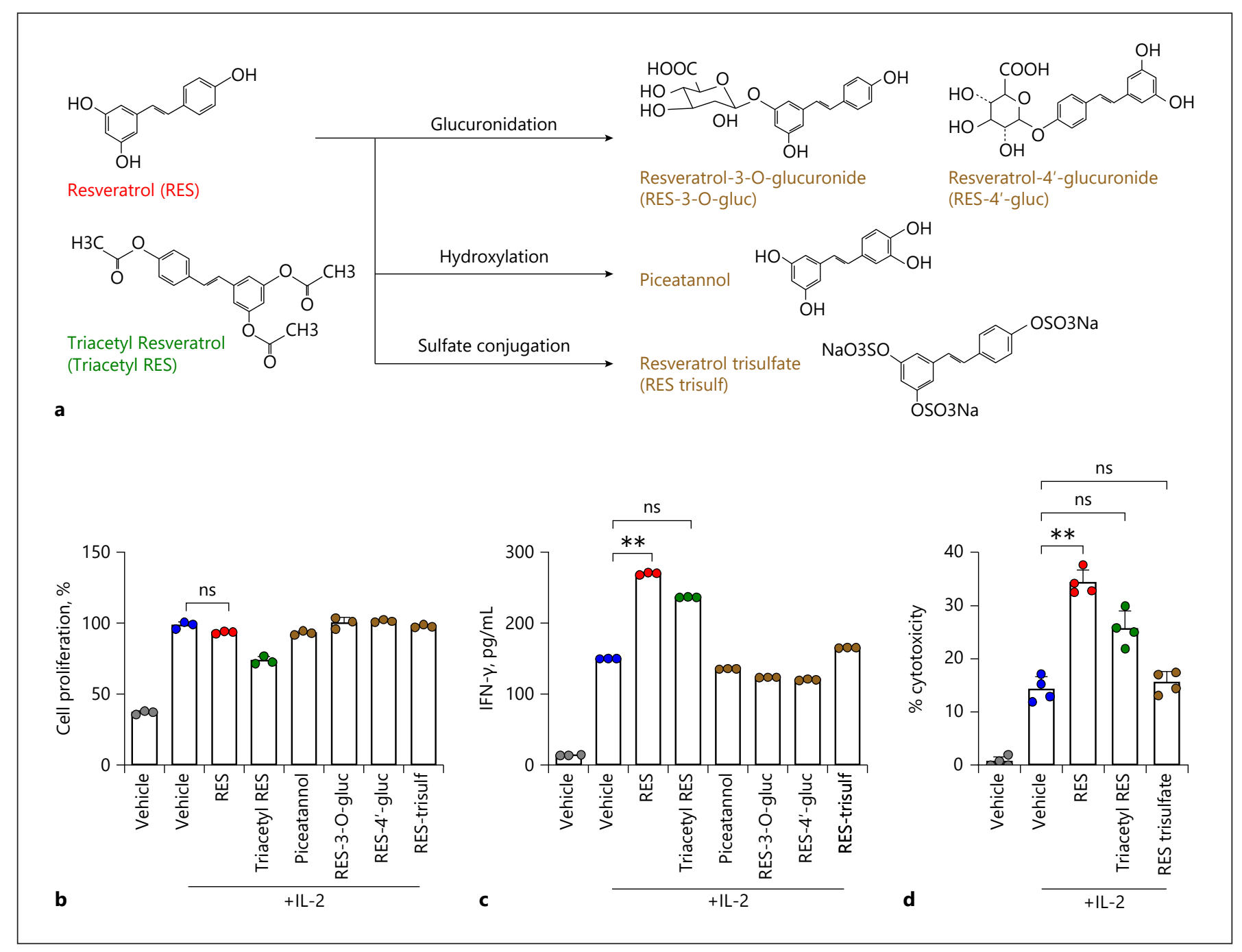

Fig. 4. Effects of derivatives and metabolites of resveratrol. a Metabolism of resveratrol and structures of resveratrol, triacetyl resveratrol (derivative of resveratrol), and metabolites of resveratrol. Resveratrol is metabolized by hydroxylation or glucuronidation or sulphate conjugation to produce combined forms of sulphate and glucuronide. b, c Cell proliferation and IFN- $\gamma$ secretion in NK92 cells after indicated treatments at $20 \mu \mathrm{M}$ (with $5 \mathrm{ng} / \mathrm{mL}$ of IL-2) for $36 \mathrm{~h}$ were determined by CCK- 8 and ELISA. Data are shown as mean \pm SEM of 3 independent experiments. $\mathbf{d}$ NK cell cytotoxicity was determined using calcein-AM. NK92 effector (E) cells were treated with $20 \mu \mathrm{M}$ resveratrol or its metabolites for $36 \mathrm{~h}$, followed by incubation with K562 target (T) cells at a 1:1 ratio ratio for $4 \mathrm{~h}$. Data are shown as mean \pm SEM of 4 independent experiments. Asterisks indicate statistical significance by one-way ANOVA: ${ }^{*} p<0.05 ;{ }^{* *} p<0.01 ;{ }^{* * *} p<0.001$; ns, not significant $(p>0.05)$. IL-2, interleukin-2; IFN- $\gamma$, interferon-gamma; RES, resveratrol.

lished after synthesis of the metabolites. Similarly, the major site of sulphate conjugation, in the $4^{\prime}$-position, was also established by synthesis (Fig. 4a) [25]. So, we investigated whether the derivative or any of the metabolites of resveratrol could activate NK cells better than resveratrol itself. First, we investigated the effects of these compounds on NK cell proliferation. Triacetyl resveratrol, a derivative of resveratrol, slightly inhibited cell proliferation, but the other compounds did not (Fig. 4b). Next, we 
measured IFN- $\gamma$ secretion in NK cells after treatment with the resveratrol derivative and metabolites. As shown in Figure 4c, the derivative and most of the metabolites did not affect IFN- $\gamma$ secretion. Triacetyl resveratrol and resveratrol trisulphate increased IFN- $\gamma$ secretion compared to that observed in IL-2-alone-treated cells, but their effect was weaker than that of resveratrol. Finally, we examined the effects of triacetyl resveratrol and resveratrol trisulphate on NK cytotoxicity using K562 target cells and the calcein-AM method. As shown in Figure 4d, after treatment with resveratrol itself, NK cytotoxicity was increased by $20 \%$ at an E:T ratio of 1:1, compared to that of IL-2 treatment alone. After triacetyl resveratrol treatment, NK cytotoxicity was increased by $11.4 \%$ at an E:T ratio of 1:1, but not significant. Unlike resveratrol and triacetyl resveratrol, resveratrol trisulphate did not affect NK cytotoxicity. These results indicate that the intact form of resveratrol is the most effective for activating NK cells.

\section{Anti-Cancer and Anti-Metastatic Effects of Resveratrol in Mice}

We examined the in vivo efficacy of resveratrol administration in melanoma mouse models. In vivo activation of NK cells by resveratrol and its anti-cancer effects were assessed by 2 different methods. First, 18 days after s.c. injection of B16F10 cells, tumour volumes were measured after dissection. As shown in Figure 5a, administration of resveratrol $(0.5 \mathrm{mg} / \mathrm{kg})$ in the presence of IL-2 significantly inhibited tumour growth compared to the administration of IL-2 alone. Quantitative analysis showed that administration of IL-2 inhibited tumour growth compared to that in vehicle control mice. Furthermore, administration of resveratrol in the presence of IL-2 significantly inhibited tumour growth compared to the administration of IL-2 alone. Treatment of resveratrol together with IL-2 significantly inhibited tumour growth (Fig. 5a) accompanied with robust activation of NK cells in the tumour (Fig. 5b) as well as draining lymph

Fig. 5. Anti-cancer and anti-metastatic effects of resveratrol in mice. B16F10 cells $\left(5 \times 10^{5}\right)$ in $0.2 \mathrm{~mL}$ PBS were injected subcutaneously into C57BL/6 mice on day $0(n=14)$. a The tumour volume was measured after dissection on day 18 after injection of melanoma cells. The activity of NK cells isolated from tumour (b) and draining lymph node (c) was evaluated by flow cytometry. B16F10 cells $\left(1 \times 10^{5}\right)$ in $0.1 \mathrm{~mL}$ PBS were injected into the tail veins of C57BL/ 6 mice on day $0(n=10)$. $\mathbf{d}$ The melanoma foci on the lung surface were counted macroscopically 18 days after injection of melanoma cells. e The activity of NK cells isolated from the lungs was evaluated by flow cytometry. f To deplete NK cells, nodes (Fig. 5c) compared to the vehicle or IL-2-treated group.

Second, we investigated whether resveratrol affects tumour metastasis in the lung metastatic model as well. B16F10 cells were i.v. injected into mice; after 18 days, the lungs were extracted, and melanoma foci on lung surfaces were counted. As shown in Figure 5d, the number of melanoma foci decreased in mice treated with resveratrol. Furthermore, IFN- $\gamma$ production and degranulation levels were significantly enhanced in the lung residual NK cells by the treatment of resveratrol compared with the vehicle or IL-2-treated group (Fig. 5e). These results suggest that resveratrol may prohibit tumour growth and metastasis by activating NK cells. Finally, to clarify the intrinsic roles of NK cells on anti-tumoural effects of resveratrol, mice were injected with tumour and treated with vehicle, resveratrol, or resveratrol plus antiNK1.1 to deplete NK cells in vivo. Intriguingly, as shown in Figure 5f, anti-tumoural effects of resveratrol was almost completely abrogated by the depletion of NK cells. Consistent with previous results, resveratrol treatment with isotype antibody significantly reduced tumour growth. However, anti-tumoural effects of resveratrol were completely abrogated upon the depletion of NK cells (Fig. 5f). Altogether, these results indicate the pivotal roles of NK cells for resveratrol-dependent anti-tumoural effects.

\section{Ex vivo Effects of Resveratrol in Human and Mouse Whole Blood}

Further, we investigated the effect of resveratrol on human and mouse NK cell activity in whole blood using the NK Vue kit. After resveratrol treatment with the activator, human NK cell activity increased by about $25 \%$ compared to treatment with the activator alone (Fig. 6a). Likewise, after resveratrol treatment with the activator, tumour-naïve mouse NK cell activity increased by about $27.2 \%$ compared to treatment with the activator alone (Fig. 6b). Also, NK cell activity was measured in tumour-

$25 \mu$ g anti-NK1.1 monoclonal antibody (PK136) or control mouse IgG2a was injected intraperitoneally (i.p.) on days $-3,2$, and 8 . B16F10 cells $\left(5 \times 10^{5}\right.$ cells $)$ in $0.2 \mathrm{~mL}$ of PBS were injected subcutaneously into the right flanks of mice on day 0 . The tumour volume was measured after dissection on day 18 after injection of melanoma cells. To evaluate the activity of resveratrol, mice were intravenously injected a total of 6 times, on days $-2,0,2,4,6$, and 8. Asterisks indicate statistical significance by one-way ANOVA: ${ }^{*} p<0.05 ;{ }^{* *} p<0.01 ;{ }^{* * *} p<0.001$; ns, not significant $(p>0.05)$. RES, resveratrol; IL-2, interleukin-2; IFN- $\gamma$, interferon-gamma; NK cells, natural killer cells.

(For figure see next page.) 

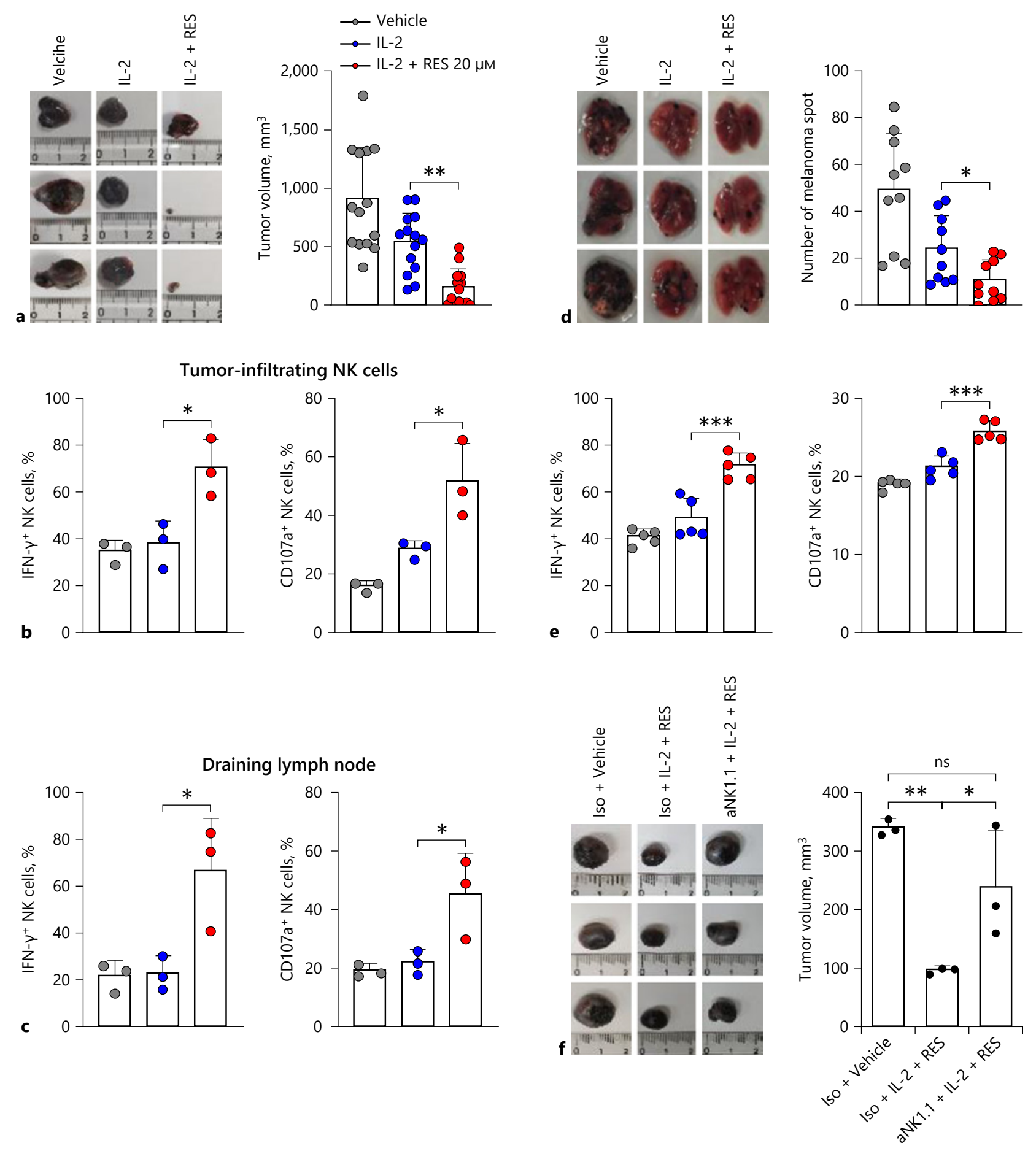

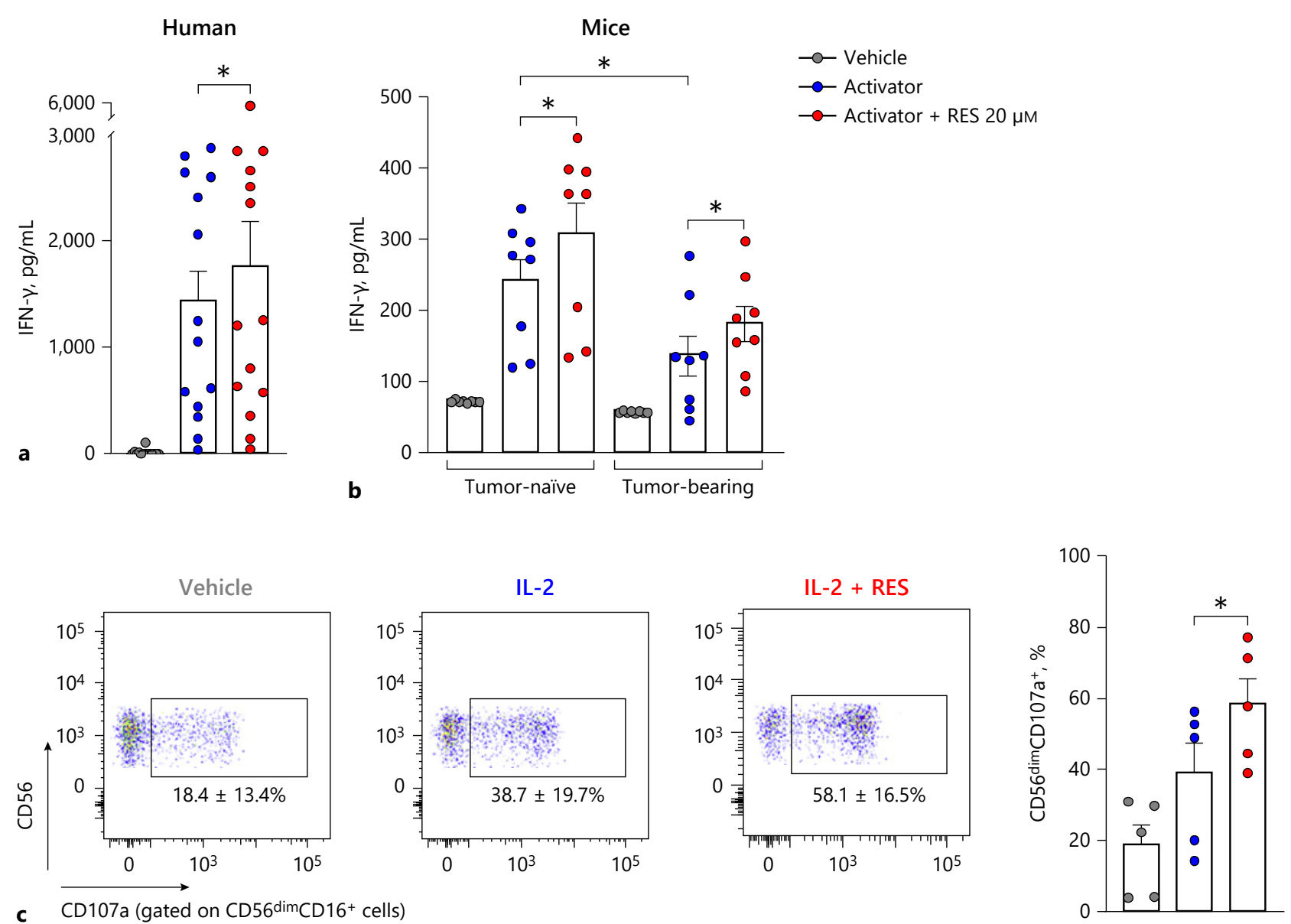

c CD107a (gated on CD56 dimCD16+ cells)

Fig. 6. Ex vivo effects of resveratrol in human and mouse whole blood. a For the human NK cell activity assay, $1 \mathrm{~mL}$ of whole blood was incubated with or without the activator and resveratrol for $24 \mathrm{~h}$ at $37^{\circ} \mathrm{C}$. b For the mouse NK cell activity assay, $100 \mu \mathrm{L}$ of whole blood was incubated with or without $30 \mu \mathrm{L}$ activator and resveratrol for $24 \mathrm{~h}$ at $37^{\circ} \mathrm{C}$ (tumour-naïve or tumour-bearing mouse model, $n=8)$. The supernatant was collected, and the released IFN- $\gamma$ was measured using the NK Vue kit. c CD107a ex- pression of primary NK cells was measured by flow cytometry. PBMCs were treated with $20 \mu \mathrm{M}$ resveratrol for $24 \mathrm{~h}$ in the presence of IL-2, followed by co-culture with K562 target cells at a 5:1 (E:T) ratio for $4 \mathrm{~h}(n=5)$. Asterisks indicate statistical significance by Dunn's multiple comparison test: ${ }^{*} p<0.05 ;{ }^{* *} p<0.01$; ${ }^{* * *} p<$ 0.001 ; ns, not significant $(p>0.05)$. IL-2, interleukin- 2 ; IFN- $\gamma$, interferon-gamma; RES, resveratrol. bearing mouse models. Even in the presence of an activator, NK cell activity was significantly lower in the tumourbearing mouse than in the tumour-naïve group (Fig. 6b). However, after resveratrol treatment in the presence of the activator, NK cell activity increased by about $27.3 \%$ compared to treatment with the activator alone in the tumour-bearing mouse (Fig. 6b). Finally, PBMCs were isolated from whole blood and treated with resveratrol for $24 \mathrm{~h}$ in the presence IL-2 and then stimulated by K562 target cells (Fig. 6c). Intriguingly, compared with IL2 -treated cells, we found that resveratrol significantly enhanced degranulation in $\mathrm{CD} 56^{\mathrm{dim}} \mathrm{CD} 16^{+}$human primary NK cells known for their cytolytic activity (Fig. 6c) [26]. Thus, these results indicate that resveratrol has potential to activate human primary NK cells and suggest that resveratrol could be used in cancer therapy by activating NK cells. 


\section{Discussion}

Resveratrol is a natural compound and antioxidant found in peanuts, soy, hop, and various fruits, especially grapes and red wines $[27,28]$. Resveratrol possesses various biological activities, including anti-tumour, anti-viral, anti-inflammation, anti-fungal, and antibacterial effects [27, 29-32]. We have demonstrated that resveratrol increases the expression of activating receptors on NK cells (Fig. 3). Interestingly, we found that resveratrol + IL-2 significantly increase NKp30 more than IL-2 alone. Especially, NKp30a and NKp30b isoforms that have immune-stimulatory roles were significantly increased by resveratrol more than IL-2 alone. One of the proposed approaches for tumour therapy is enhancing tumour sensitivity to NK cell cytolysis by upregulating the NKp30 ligand B7-H6 on tumour cells using stress inducers [33]. Moreover, preclinical approaches involve increasing tumour sensitivity to NK cells by upregulating the expression of NKG2D ligands on cancer cells using HDAC inhibitors [34]. Enhancement of cell surface expression of NKG2D ligands and DR4/5 on various cancer cells by resveratrol increases TRAIL sensitivity and could facilitate NK cell-mediated killing of cancer cells $[35,36]$. However, there are no reports that resveratrol also increases B7-H6 expression on cancer cells. If resveratrol did induce the expression of $\mathrm{B} 7-\mathrm{H} 6$ on cancer cells, it would be a good candidate for an anti-cancer drug, acting as both an immune stimulator for NK cells and a stress inducer for cancer cells.

NK cells require IL-2 to proliferate, and resveratrol treatment without IL-2 did not affect IFN- $\gamma$ secretion or NK cytotoxicity in vitro (Fig. 2). IL-2 is capable of activating NK cells and is used as an anti-cancer drug in immunotherapy [37]. According to a recent report, resveratrol has shown promising results in adjuvant therapy [27]. Resveratrol in combination with 5-fluoruracyl or cisplatin exhibited synergistic effects in the induction of apoptosis in cancer cells in vitro and in vivo $[38,39]$. Therefore, resveratrol can be expected to exert a greater effect in combination with IL-2.

Resveratrol shows a concentration-dependent biphasic effect [40]. Low doses of resveratrol reportedly activate NK cells, while high doses reportedly promote cell apoptosis via the caspase signalling pathway [40]. In one study, NK cell killing activity was enhanced at low concentrations of resveratrol ranging from 0.33 to $5.48 \mu \mathrm{M}$, with a maximum activity at $1.31 \mu \mathrm{M}$; however, NK cell killing activity was inhibited at higher concentrations of 21.92 and $87.68 \mu \mathrm{M}$ resveratrol [41]. In another study by $\mathrm{Lu}$ and
Chen [42], $12.5 \mu \mathrm{M}$ resveratrol induced the highest NK cell killing activity. In our study, resveratrol did not significantly affect NK cell proliferation in vitro, but it did increase IFN- $\gamma$ secretion in a dose-dependent manner up to a concentration of $20 \mu \mathrm{M}$. We also found that resveratrol increased IFN- $\gamma$ secretion ex vivo at a concentration of $20 \mu \mathrm{M}$ and did not affect survival when injected into mice at $0.5 \mathrm{mg} / \mathrm{kg}$ (data not shown).

Resveratrol is currently being sold as a dietary supplement. Resveratrol is first detectable in plasma $15 \mathrm{~min}$ after oral administration, reaching its peak concentration after $30 \mathrm{~min}$, and it is quickly metabolized in the intestine and liver into glucuronide and sulphate conjugates [43]. According to a study on the absorption of wine-related polyphenols, the highest serum level of total resveratrol (free and conjugated) was recorded after consumption of 25-mg trans-resveratrol per $70 \mathrm{~kg}$ body weight in 3 different matrices (wine, grape juice, and vegetable juice) [44]. Free trans-resveratrol accounted for $1.7-1.9 \%$ of the peak total serum concentration. This is considerably lower than the concentrations tested in vitro showing beneficial effects. Moreover, resveratrol is rapidly metabolized in the intestine by phase II enzymes, so it is very difficult to reach pharmacologically effective systemic plasma concentrations, which might significantly restrict the bioavailability of resveratrol $[45,46]$. Our data showed that IFN- $\gamma$ secretion increased upon treatment with intact resveratrol but not with its metabolites (Fig. 4). Accordingly, resveratrol would be more effective when administered by injection rather than by diet.

\section{Acknowledgements}

The authors would like to thank Si-Hwan Ko (Yonsei University College of Medicine) for his technical assistance.

\section{Statement of Ethics}

Blood was obtained from healthy donors after acquiring institutional review board approval and informed consent (No. 4-20190007). Animal experiments were approved by the Yonsei University Health System Institutional Animal Care and Use Committee (YUHS-IACUC).

\section{Conflict of Interest Statement}

The authors declare that they have no conflicts of interest. 


\section{Funding Sources}

This study was supported in part by the National Research Foundation of Korea (NRF) Grant (2016-31-0446) funded by the Korean government (MEST).

\section{Author Contributions}

J. Kim: conceptualization, design, supervision, project administration, funding acquisition, interpretation of data, and final approval of the manuscript; Y. Lee: conceptualization, design, investigation, interpretation of data, validation, visualization, and manuscript writing; H. Shin: design and interpretation of data.

\section{References}

1 Cerwenka A, Lanier LL. Natural killer cells, viruses and cancer. Nat Rev Immunol. 2001 Oct; $1(1): 41-9$.

2 Vivier E, Tomasello E, Baratin M, Walzer T, Ugolini S. Functions of natural killer cells. Nat Immunol. 2008 May;9(5):503-10.

3 Guillerey C, Huntington ND, Smyth MJ. Targeting natural killer cells in cancer immunotherapy. Nat Immunol. 2016 Aug 19;17(9): 1025-36.

4 Jung YS, Park JH, Park DI, Sohn CI, Lee JM, Kim TI. Physical inactivity and unhealthy metabolic status are associated with decreased natural killer cell activity. Yonsei Med J. 2018 Jun;59(4):554-62.

5 Kim HR, Lee KH, Park SJ, Kim SY, Yang YK, Tae J, et al. Anti-cancer activity and mechanistic features of a NK cell activating molecule. Cancer Immunol Immunother. 2009 Oct;58(10):1691-700.

6 Glasner A, Ghadially H, Gur C, Stanietsky N, Tsukerman P, Enk J, et al. Recognition and prevention of tumor metastasis by the NK receptor NKp46/NCR1. J Immunol. 2012 Mar 15;188(6):2509-15.

7 Morvan MG, Lanier LL. NK cells and cancer: you can teach innate cells new tricks. Nat Rev Cancer. 2016 Jan;16(1):7-19.

8 Zwirner NW, Domaica CI. Cytokine regulation of natural killer cell effector functions. Biofactors. 2010 Jul-Aug;36(4):274-88.

9 Koch J, Steinle A, Watzl C, Mandelboim O. Activating natural cytotoxicity receptors of natural killer cells in cancer and infection. Trends Immunol. 2013 Apr;34(4):182-91.

10 Abel AM, Yang C, Thakar MS, Malarkannan S. Natural killer cells: development, maturation, and clinical utilization. Front Immunol. 2018;9:1869.

11 Smyth MJ, Hayakawa Y, Takeda K, Yagita H New aspects of natural-killer-cell surveillance and therapy of cancer. Nat Rev Cancer. 2002 Nov;2(11):850-61.

12 Wang R, Jaw JJ, Stutzman NC, Zou Z, Sun PD Natural killer cell-produced IFN- $\gamma$ and TNF- $\alpha$ induce target cell cytolysis through up-regulation of ICAM-1. J Leukoc Biol. 2012 Feb;91(2):299-309.

13 Vivier E, Raulet DH, Moretta A, Caligiuri MA Zitvogel L, Lanier LL, et al. Innate or adaptive immunity? The example of natural killer cells. Science. 2011 Jan 7;331(6013):44-9.

14 Dempke WCM, Fenchel K, Uciechowski P, Dale SP. Second- and third-generation drugs for immuno-oncology treatment: the more the better? Eur J Cancer. 2017 Mar;74:55-72.
15 Sharma P, Hu-Lieskovan S, Wargo JA, Ribas A. Primary, adaptive, and acquired resistance to cancer immunotherapy. Cell. 2017 Feb 9; 168(4):707-23.

16 Chiossone L, Dumas PY, Vienne M, Vivier E. Natural killer cells and other innate lymphoid cells in cancer. Nat Rev Immunol. 2018 Nov; 18(11):671-88.

17 Van Schandevyl S, Kerre T. Chimeric antigen receptor T-cell therapy: design improvements and therapeutic strategies in cancer treatment. Acta Clin Belg. 2018 Nov 13;75(1):26-32.

18 Buzio C, Andrulli S, Santi R, Pavone L, Passalacqua R, Potenzoni D, et al. Long-term immunotherapy with low-dose interleukin-2 and interferon-alpha in the treatment of patients with advanced renal cell carcinoma. Cancer. 2001 Nov 1;92(9):2286-96.

19 Szczepanski MJ, Szajnik M, Welsh A, Foon KA, Whiteside TL, Boyiadzis M. Interleukin-15 enhances natural killer cell cytotoxicity in patients with acute myeloid leukemia by upregulating the activating NK cell receptors. Cancer Immunol Immunother. 2010 Jan;59(1):73-9.

20 Zhu EF, Gai SA, Opel CF, Kwan BH, Surana $\mathrm{R}, \mathrm{Mihm}$ MC, et al. Synergistic innate and adaptive immune response to combination immunotherapy with anti-tumor antigen antibodies and extended serum half-life IL-2. Cancer Cell. 2015 Apr 13;27(4):489-501.

21 Khuda-Bukhsh AR, Das S, Saha SK. Molecular approaches toward targeted cancer prevention with some food plants and their products: inflammatory and other signal pathways. Nutr Cancer. 2014;66(2):194-205.

22 Alter G, Malenfant JM, Altfeld M. CD107a as a functional marker for the identification of natural killer cell activity. J Immunol Methods. 2004 Nov;294(1-2):15-22.

23 Lozzio BB, Lozzio CB. Properties and usefulness of the original K-562 human myelogenous leukemia cell line. Leuk Res. 1979;3(6): 363-70.

24 Delahaye NF, Rusakiewicz S, Martins I, Ménard C, Roux S, Lyonnet L, et al. Alternatively spliced NKp30 isoforms affect the prognosis of gastrointestinal stromal tumors. Nat Med. 2011 Jun;17(6):700-7.

25 Walle T. Bioavailability of resveratrol. Ann N Y Acad Sci. 2011 Jan;1215:9-15.

26 De Maria A, Bozzano F, Cantoni C, Moretta L. Revisiting human natural killer cell subset function revealed cytolytic CD56(dim) CD16+ NK cells as rapid producers of abundant IFN-gamma on activation. Proc Natl Acad Sci U S A. 2011 Jan 11;108(2):728-32.
27 Varoni EM, Lo Faro AF, Sharifi-Rad J, Iriti M. Anticancer molecular mechanisms of resveratrol. Front Nutr. 2016;3:8.

28 Wiciński M, Socha M, Walczak M, Wódkiewicz E, Malinowski B, Rewerski S, et al. Beneficial effects of resveratrol administrationfocus on potential biochemical mechanisms in cardiovascular conditions. Nutrients. 2018 Nov 21;10(11):1813.

29 Kundu JK, Surh YJ. Cancer chemopreventive and therapeutic potential of resveratrol: mechanistic perspectives. Cancer Lett. 2008 Oct 8;269(2):243-61.

30 Lu CC, Lai HC, Hsieh SC, Chen JK. Resveratrol ameliorates Serratia marcescens-induced acute pneumonia in rats. J Leukoc Biol. 2008 Apr;83(4):1028-37.

31 Wang XL, Li T, Li JH, Miao SY, Xiao XZ. The effects of resveratrol on inflammation and oxidative stress in a rat model of chronic obstructive pulmonary disease. Molecules. 2017 Sep 12;22(9):1529.

32 Zhao X, Tong W, Song X, Jia R, Li L, Zou Y, et al. Antiviral effect of resveratrol in piglets infected with virulent pseudorabies virus. Viruses. 2018 Aug 27;10(9):457.

33 Cao G, Wang J, Zheng X, Wei H, Tian Z, Sun R. Tumor therapeutics work as stress inducers to enhance tumor sensitivity to natural killer (NK) cell cytolysis by up-regulating NKp30 ligand B7-H6. J Biol Chem. 2015 Dec 11; 290(50):29964-73.

34 Spear P, Wu MR, Sentman ML, Sentman CL. NKG2D ligands as therapeutic targets. Cancer Immun. 2013:13:8.

35 Fulda S, Debatin KM. Resveratrol-mediated sensitisation to TRAIL-induced apoptosis depends on death receptor and mitochondrial signalling. Eur J Cancer. 2005 Mar;41(5):786-98.

$36 \mathrm{Hu}$ L, Cao D, Li Y, He Y, Guo K. Resveratrol sensitized leukemia stem cell-like KG-1a cells to cytokine-induced killer cells-mediated cytolysis through NKG2D ligands and TRAIL receptors. Cancer Biol Ther. 2012 May;13(7):516-26.

37 Burns LJ, Weisdorf DJ, DeFor TE, Vesole DH Repka TL, Blazar BR, et al. IL-2-based immunotherapy after autologous transplantation for lymphoma and breast cancer induces immune activation and cytokine release: a phase I/II trial. Bone Marrow Transplant. 2003 Jul; 32(2):177-86

38 Cosco D, Paolino D, Maiuolo J, Marzio LD, Carafa M, Ventura CA, et al. Ultradeformable liposomes as multidrug carrier of resveratrol and 5-fluorouracil for their topical delivery. Int J Pharm. 2015 Jul 15;489(1-2):1-10. 
39 Ma L, Li W, Wang R, Nan Y, Wang Q, Liu W, et al. Resveratrol enhanced anticancer effects of cisplatin on non-small cell lung cancer cell lines by inducing mitochondrial dysfunction and cell apoptosis. Int J Oncol. 2015 Oct; 47(4):1460-8.

40 Li Q, Huyan T, Ye LJ, Li J, Shi JL, Huang QS. Concentration-dependent biphasic effects of resveratrol on human natural killer cells in vitro. J Agric Food Chem. 2014 Nov 12;62(45): 10928-35.
41 Falchetti R, Fuggetta MP, Lanzilli G, Tricarico M, Ravagnan G. Effects of resveratrol on human immune cell function. Life Sci. 2001 Nov 21;70(1):81-96.

$42 \mathrm{Lu}$ CC, Chen JK. Resveratrol enhances perforin expression and NK cell cytotoxicity through NKG2D-dependent pathways. J Cell Physiol. 2010 May;223(2):343-51.

43 Lee AM, Shandala T, Nguyen L, Muhlhausler BS, Chen KM, Howe PR, et al. Effects of resveratrol supplementation on bone growth in young rats and microarchitecture and remodeling in ageing rats. Nutrients. 2014 Dec 16; 6(12):5871-87.
44 Goldberg DM, Yan J, Soleas GJ. Absorption of three wine-related polyphenols in three different matrices by healthy subjects. Clin Biochem. 2003 Feb;36(1):79-87.

45 Wenzel E, Somoza V. Metabolism and bioavailability of trans-resveratrol. Mol Nutr Food Res. 2005 May;49(5):472-81.

46 Leischner C, Burkard M, Pfeiffer MM, Lauer UM, Busch C, Venturelli S. Nutritional immunology: function of natural killer cells and their modulation by resveratrol for cancer prevention and treatment. Nutr J. 2016 May 4;15(1):47. 